\title{
El impacto del sistema de aseguramiento de la calidad en el servicio entregado por las universidades privadas en Chile
}

\author{
The impact of the quality assurance system in the service \\ delivered by the private universities in Chile \\ Emilio Rodríguez-Ponce ${ }^{1} \quad$ Liliana Pedraja-Rejas $^{1} \quad$ Carmen Araneda-Guirriman $^{1}$ \\ María González-Plitt ${ }^{1} \quad$ Juan Rodríguez-Ponce ${ }^{1}$ \\ Recibido 9 de junio de 2011, aceptado 9 de noviembre de 2011 \\ Received: June 9, 2011 Accepted: November 9, 2011
}

\begin{abstract}
RESUMEN
Esta investigación analiza la relación entre los sistemas de aseguramiento de la calidad de las universidades y el servicio entregado por éstas. Para este efecto se desarrolla un marco conceptual que vincula ambas variables, y posteriormente se analiza la pertinencia de la hipótesis planteada trabajando con una muestra de veinte universidades privadas que han participado de procesos de acreditación institucional.

Los resultados muestran que el sistema de aseguramiento de la calidad, medido por los años de acreditación, tiene una capacidad explicativa del $41,5 \%$ de la calidad del servicio en las universidades analizadas.

Por lo tanto, se concluye que los procesos de aseguramiento de la calidad de las instituciones de educación terciaria son un elemento constitutivo y determinante de la calidad del servicio de estas instituciones.

Palabras clave: Calidad, calidad de servicio, aseguramiento de la calidad, educación superior, universidades privadas.
\end{abstract}

\section{ABSTRACT}

This study examines the relationship between quality assurance systems of universities and the service provided by them. For this purpose, we develop a conceptual framework that links the two variables and then discusses the relevance of the hypothesis to work with a sample of twenty private universities that have participated in processes of institutional accreditation.

The results show that the system of quality assurance, as measured by the years of accreditation, has explanatory power of $41.5 \%$ service quality in universities analyzed.

Therefore, we conclude that the processes of quality assurance of tertiary education institutions, are a constituent and determinant of the quality of service of these institutions.

Keywords: Quality, quality service, quality assurance, higher education, private universities.

\section{INTRODUCCIÓN}

La globalización ha forjado transformaciones en escalas similares en aspectos relacionados a cómo la gente vive, trabaja, identifican y agregan, comunican y cómo ellos se interconectan local, nacional, internacional y globalmente; cómo ellos están siendo educados, incluyendo cambios en la naturaleza misma del Estado. La globalización hace alusión a los intercambios dinámicos en el mundo contemporáneo.

En el centro de la globalización están las transformaciones en cómo el capital circula en todo el mundo y es vinculado a la producción y consumo [28].

\footnotetext{
1 Universidad de Tarapacá. 18 de Septiembre 2222. Arica, Chile. E-mail: erodrigu@uta.cl; lpedraja@uta.cl; caraneda@uta.cl; jrodrigup@uta.cl
} 
En el aspecto social, desde la primera infancia hasta el postgrado, la educación se encuentra en medio de un complejo proceso de cambio. En este contexto la educación siempre entrega una noción de cómo el mundo funciona y debiera funcionar. A través de los procesos educacionales se busca organizar y convenir con otros, particularmente con la nueva generación, el ser colectivo, el mundo en que se vive, las aspiraciones, los valores y la sabiduría [28]. En este escenario surgen en todos los países desafíos para las universidades [13].

Las instituciones educacionales se encuentran con la presión de responder rápidamente a los cambios en los entornos sociales, armados con recursos que son insuficientes [28], debiendo velar al mismo tiempo por la calidad y pertinencia de su oferta académica [35].

Las Instituciones de Educación Superior deben responder a las diversas necesidades y requerimientos del entorno, siendo capaces de ajustarse a los constantes cambios.

Asimismo, es importante consignar que el sistema de Educación Superior ha sufrido diversos cambios, entre los cuales se encuentra su rápido crecimiento, necesitando a causa de esto autoexaminarse para poder ver si su calidad está siendo mantenida [33]. La calidad es, por ende, un factor fundamental a ser considerado en las Instituciones de Educación Superior [15].

En este sentido, el aseguramiento de la calidad es visto como un proceso que traslapa la planificación estratégica de las instituciones, pues es una actividad de gestión donde la alta calidad puede ser esperada. De esta forma, el sistema de aseguramiento de la calidad puede ser descrito usando el concepto de mapa de calidad, el cual es una representación visual de cómo el entorno es incorporado en una planificación estratégica [24].

Aquellas Instituciones de Educación Superior, donde el sistema es afectivo y la administración es servicial, dispuestos siempre a entregar servicios de calidad pueden disfrutar del ingreso de estudiantes brillantes y talentosos [26].

De esta manera resulta fundamental velar por la calidad de servicio en la Educación Superior, ya que ésta repercute directamente en la percepción que tienen los estudiantes, siendo uno de los factores determinantes al momento de decidir en qué Institución de Educación Superior estudiar.

Por ende, el reto de la gestión de calidad se basa en formular patrones que puedan ser evaluados, contrastando el quehacer real de cada universidad con las exigencias de calidad, y buscando un proceso de mejoramiento constante [38].

Cabe consignar que la calidad es multidimensional y como tal debe alcanzar todas las funciones $\mathrm{y}$ actividades que conlleva el quehacer de las universidades [41].

En la literatura revisada se releva la importancia que tienen los procesos de aseguramiento de la calidad en las Instituciones de Educación Superior, no obstante falta mayor evidencia empírica que demuestre cómo estos procesos repercuten en la calidad de servicio de las universidades, por ende se visualiza una instancia para analizar dicho impacto y vinculación.

Esta investigación tiene como propósito analizar el impacto del aseguramiento de la calidad institucional sobre la calidad de servicio de las Instituciones de Educación Superior, efectuando un aporte al conjunto de conocimientos, entregando evidencia empírica de la magnitud de esta relación en el caso particular de las universidades privadas chilenas.

\section{MARCO TEÓRICO}

Actualmente, la demanda por Educación Superior se ha visto incrementada en el mundo entero y la respuesta a esto ha sido una expansión de la oferta universitaria tanto al interior de los países como entre éstos [10].

En este sentido, las universidades se han visto permanentemente afectadas por las tendencias internacionales y, en cierto grado, funcionan más allá de una gran variedad de instituciones académicas de la comunidad internacional. Teniendo, por ende, la globalización una fuerte influencia en la Educación Superior [5].

A esto se suma el requerimiento constante que se les hace a las Instituciones de Educación Superior 
para que demuestren procesos y resultados que den certeza del cumplimiento de los patrones de calidad tanto a nivel nacional como global [35], ya que todas las universidades alrededor del mundo tratan de convertirse en universidades de clase mundial [22].

Cabe consignar además que la Educación Superior tiene un rol en la sociedad y cultura contemporánea vinculado a dos procesos paralelos, primero es el cuestionamiento del rol del Estado Nación en la era global y la gradual descomposición del Estado de Bienestar en la mayoría de los países de la OECD [25].

Es al interior de este contexto donde el aseguramiento de la calidad en las Instituciones de Educación Superior ha alcanzado el punto más alto de la agenda política en muchas naciones [5].

Por ende, la calidad es un aspecto fundamental al interior de la Educación Superior, ya que tanto los estudiantes como la sociedad en su conjunto requieren una formación profesional que responda y se adecue a las necesidades del entorno laboral y social. Por lo tanto, las universidades deben enfrentar el asunto de la calidad como una forma de alcanzar esta meta, focalizándose hacia los clientes, considerando las interacciones entre los sujetos como un elemento sustancial de la calidad del servicio [34].

A este respecto es importante señalar que la calidad es un concepto multidimensional, cuyos estándares para evaluar la Educación Superior han sido establecidos en gran parte del mundo. Con relación a esto, un hecho que marca un quiebre con el pasado son las nuevas tendencias que hacen que el proceso sea más dependiente de sus pares que de las autoridades gubernamentales. Además, las instituciones son más frecuentemente evaluadas contra su propia misión, que frente a un modelo institucional definido por una agencia reguladora [5].

En consecuencia, actualmente se puede hablar de un mercado de consumidores, particularmente en el caso del pregrado con una extensa libertad de elección por parte de los alumnos que pagan por el servicio, ya sea de manera directa o por medio de becas y créditos (con diversos grados de subsidios) financiados por el gobierno. Existe además, en este escenario, el funcionamiento de un mercado de prestigios institucionales, donde la reputación de las instituciones operaría como una señal de calidad, generando la atracción de los mejores estudiantes y los profesores de mayor prestigio, en una espiral que bajo condiciones del mercado tiende a retroalimentarse permanentemente con consecuencias de autorreforzamiento [10].

Por esta razón, es fundamental profundizar en el análisis de la relación y en el impacto que tienen los procesos de aseguramiento de la calidad, en la calidad de los servicios entregados al interior de las universidades privadas de Chile.

\section{Aseguramiento de la calidad en la educación superior}

Al hablar de aseguramiento de la calidad es importante distinguir este término respecto a otros relativos a calidad. De esta forma, nos encontramos con conceptos tales como: evaluación de calidad, control de calidad, auditoría de calidad, administración total de calidad. La evaluación de calidad está referida al proceso de evaluación externa de la calidad de la enseñanza y el aprendizaje en la Educación Superior [32]. El control de calidad es un término utilizado para describir las técnicas operacionales o procedimientos sistemáticos [6]. Auditoría de calidad se entiende como el proceso que garantiza el control de calidad acordado en una institución sea satisfactorio [32]. Este concepto hace referencia, por ende, a las políticas, sistemas y procesos dirigidos a garantizar el mantenimiento y mejoramiento de la calidad en la Educación Superior [23]. De esta forma, al ser declarado en la misión de una universidad, refleja de cierto modo la cultura de calidad que la institución se esfuerza por implementar, donde los programas deben ser regularmente ajustados a las necesidades de los estudiantes y empleadores. Las instituciones deben responder a las oportunidades de los procesos de enseñanza y aprendizaje sobre bases regulares [7].

Asimismo, se configura como un espacio contradictorio, donde coexisten dentro de un mismo proyecto: identidad, derechos y regulación. Siendo representado como una intervención liberadora, promoviendo el empoderamiento del consumidor y el cuidado del cliente [27].

En este sentido, el tema del aseguramiento de la calidad está siendo una prioridad en los procesos de convergencia del Área Europea de Educación 
Superior, donde la mayoría de las universidades se encuentran trabajando estos temas, donde es muy fuerte la idea de que estos procesos son un motor de cambio de las Instituciones de Educación Superior [18].

Efectivamente, los procesos orientados a resguardar la calidad se han transformado en un fenómeno transnacional, siendo el Proceso Bolonia en Europa la transformación multinacional más importante hasta ahora, teniendo una garantía de calidad ejecutada por medio de organizaciones multinacionales [19].

De este modo, se convierte la calidad en parte integral de la Educación Superior [37].

Por otra parte, es importante señalar otros conceptos asociados a calidad, como lo es el término fitness for purpose, el cual considera en primera instancia la definición de un propósito y el establecimiento de un criterio con el que se pueda decidir qué se puede hacer. La definición del criterio es mucho más fácil para la industria de manufacturas que para la educación [16]. Las personas han hecho intentos inapropiados, inadecuados e impensados para aplicar metodologías provenientes de las industrias, a las universidades y escuelas, generando algunas veces, con razón, la molestia de investigadores, profesores e investigadores [16].

Asimismo, dos palabras hacen la diferencia entre Fitness tolfor y Fitness of. El modelo fitness to/ for provee el propósito de la calidad, define un currículum con objetivos de aprendizajes, resultados adecuados al logro de los propósitos y la construcción de un conjunto de constructos como criterios de evaluación para asegurar que el alumno ha logrado por lo menos el contenido mínimo suficiente de los objetivos o resultados para que le sea concedido el grado [16]. Pueden existir diversos propósitos, pero todo sistema de aseguramiento de la calidad debe asegurarse de tener uno. Estos propósitos podrían estar restringidos a resultados materiales u otros más bien cualitativos, como sucede en los procesos de enseñanza [16].

En el modelo de fitness of purpose el proceso es el mismo, salvaguarda el contenido del currículum, resultados, entre otros. No obstante, ha sido además contrastado con algunos puntos de referencia, criterios y estándares externos. Estas aplicaciones son más difíciles para las experiencias de aprendizaje [16].

El aseguramiento de la calidad es algo que las Instituciones de Educación Superior deben hacer, teniendo que contar para ello con una metodología que permita juzgar la calidad con la cual los macro y microobjetivos organizacionales y los resultados han sido alcanzados [16].

Es necesario establecer, por lo tanto, mecanismos de aseguramiento de la calidad, que den garantía pública sobre el cumplimiento de los objetivos declarados, se otorgue la información adecuada y suficiente para la toma de decisiones, se satisfagan las demandas de los usuarios y finalmente es necesario que existan mecanismos para que las Instituciones de Educación Superior rindan cuenta pública de su quehacer [12].

Las Instituciones de Educación Superior al tener mecanismos que resguarden la calidad, en términos generales, se aseguran de tener una buena calidad de servicio, siendo más importante aún el reconocimiento y la percepción positiva de este hecho por parte de los clientes.

Los directivos académicos y los académicos emprendedores de las universidades deben permanecer dentro del sector universitario, involucrándose activamente en las transformaciones que está sufriendo la educación al interior de la cultura de negocios vigentes. Para esto, deben fomentar cambios tales como establecer medidas de control del aseguramiento de la calidad para estar en la redefinición de procesos. Los procedimientos de aseguramiento de la calidad han necesitado la división de complejos procesos intelectuales, transformando competencias y habilidades a niveles medibles, los cuales son sólo comparables en un nivel general amplio [17].

Los procesos de aseguramiento de la calidad dan particular atención a los procedimientos institucionales para la evaluación de los estudiantes. Los procesos de evaluación de los estudiantes deben tener criterios claros y explícitos de calificación [14].

\section{Calidad del servicio}

Hoy en día la competitividad en el medio académico se traduce en más opciones disponibles para 
los estudiantes. Esto hace que las instituciones educacionales estén permanentemente preocupadas de atraer y retener a los estudiantes [20]. Siendo la calidad un mecanismo clave para la atracción y retención de alumnos.

A este respecto, las primeras investigaciones sobre calidad del servicio en Educación Superior enfatizan frecuentemente el aspecto académico por sobre el administrativo, concentrándose en eficaces mecanismos de entrega y la calidad de los cursos y la enseñanza [20]. Siendo este último casi tan importante para los clientes al momento de evaluar la calidad del servicio entregado por las instituciones de Educación Superior.

La calidad del servicio es también vista como un prerrequisito para establecer y sustentar una relación satisfactoria con el cliente. La relación entre calidad de servicio y satisfacción de clientes ha surgido como un asunto y tema estratégico para las instituciones. La calidad de servicio es considerada como un antecedente a la satisfacción [20].

Cabe consignar que la calidad en la Educación Superior es fenómeno complejo y multifacético, siendo insuficiente contar sólo con una definición de calidad; no existe, por ende, una sola forma de definir y medir la calidad de servicio. Cada uno de los actores involucrados en la Educación Superior tiene una mirada particular de la calidad de la educación (gobierno, estudiantes, personal académico) dependiendo ésta de sus propias necesidades [39].

Para mantener la calidad e incrementar la rendición de cuentas, las Instituciones de Educación Superior deben esforzarse en desarrollar procedimientos alternativos de evaluación [21].

Resultados encontrados sugieren que para una calidad efectiva en la Educación Superior se debe ir más allá de las ideas tradicionales, para que así refleje las necesidades y realidades locales dentro de un contexto internacional, conducido por un monitoreo eficiente de la calidad y mecanismos de evaluación [3].

Por ende, para mejorar la calidad de los servicios de los clientes se deben conocer y comprender sus necesidades, una vez hecho esto se deben acoger los atributos de calidad acogidos por los clientes [11].

La calidad en la Educación Superior, en términos generales, puede ser entendida entonces como el grado de ajuste entre las acciones que una institución, programa académico o carrera ejecuta para cumplir a las orientaciones que surgen desde su misión y propósitos institucionales y resultados que de éstas consigue [12]. Involucrando además las necesidades de los diversos actores inmersos en los procesos de calidad en la Educación Superior.

\section{La relación entre aseguramiento de la calidad y la calidad del servicio}

En relación a lo señalado anteriormente, la medición de la calidad del servicio en la Educación Superior es cada día más importante para atraer y retener las matrículas basadas en ingresos. La calidad ha sido descrita como unidades de calidad agrupadas en un producto o servicio [4].

El estudio de la calidad del servicio ha sido acaloradamente debatido en la literatura del marketing por más de dos décadas. Siendo entendida como una medida de cuán bien el nivel del servicio entregado responde a las expectativas del cliente [31].

La educación es uno de los servicios más intangibles de todos [36], explicaron cómo ha sido el intento por aproximar el tema del aseguramiento de la calidad, particularmente en la industria de servicio, desde el punto de vista del estudiante.

El principio de marketing, el cual sugiere que la estrategia corporativa debiera fluir desde las necesidades de los consumidores, no ha tenido mucho crédito en las discusiones sobre rendición de cuentas de las universidades. Se ha sugerido que existe en la literatura evidencia sobre el impacto de la calidad de servicio en la educación superior, lo cual se ha dado desde que la calidad del servicio se transformó en uno de los mayores insumos para la Educación Superior [21].

Las industrias de servicio están experimentando un incremento importante en su rol sobre la economía, siendo actualmente clave para la sobrevivencia y éxito, considerada como una tendencia de gran poder competitivo. Además es un elemento altamente 
estratégico a ser considerado en la agenda directiva [21].

Cabe señalar que los estudios que han medido la calidad del servicio han utilizado como metodología de medición las escalas de evaluación. Algunas de estas derivan de la realización de modelos conceptuales generados al entender los procesos de evaluación.

Un estudio de calidad de Servicio en Educación Superior combinó dos escalas, HEdPERF-SERVPERF, emergiendo una nueva estructura dimensional de calidad del servicio a partir de los ítems de ambas, encontrándose en los resultados cuatro factores que se enuncian a continuación: Factor 1, Aspectos no Académicos, variables esenciales para permitir a los estudiantes cumplir con sus obligaciones y deberes estudiantiles. Hace referencia a la capacidad y voluntad de la administración de mostrar respeto, igualdad de trato y confidencialidad de la información. Factor 2, Aspectos Académicos, representa la responsabilidad de los académicos y poner en valor atributos tales como una actitud positiva, buena comunicación. Estos elementos se centran en la reputación académica de la institución. Factor 3, Fiabilidad, consiste en dar énfasis a la capacidad de proveer los servicios comprometidos sobre el tiempo exacto y con seguridad, y por último el Factor 4, Empatía, este factor se relaciona con la entrega de atención individualizada y personalizada a los estudiantes, con un claro entendimiento de sus necesidades específicas y crecientes [1].

Actualmente, las universidades tienen que asegurar sus productos y servicios, como los elementos descritos anteriormente. Al interior de las Instituciones de Educación Superior está la gestión de bienes, servicios y actividades desde una fase de insumos, desarrollo de procesos, hasta la fase de salida de la producción. Los procesos de aseguramiento de la calidad reconocen la necesidad de la universidad de aceptar la responsabilidad de sus propios procesos de gestión. Por tanto, el aseguramiento de la calidad es un proceso total, holístico, orientado a asegurar la integridad de sus resultados [30].

Es así como el aseguramiento de la calidad puede ser descrito como todos los planes y sistemáticas actividades que entregan adecuada confianza de que el producto o servicio está satisfaciendo determinados requisitos de calidad [34].
En este sentido, podemos señalar que el aseguramiento de la calidad es un término holístico, el cual es dirigido hacia la educación como una organización. Conlleva elementos tales como los proveedores y consumidores y diversas actividades puestas en un lugar para generar productos y servicios [29].

\section{METODOLOGÍA}

La metodología del estudio es cuantitativa, de orden transversal, además es carácter exploratorio y descriptivo.

\section{Muestra}

Se utilizó una muestra no probabilística y no representativa de 20 universidades privadas chilenas que han participado en los procesos de acreditación institucional conducidos por la Comisión Nacional de Acreditación de Chile, las cuales tenían además registros de reclamos formales en el Servicio Nacional del Consumidor.

El criterio de selección de las universidades estuvo determinado por contar con los dos criterios señalados anteriormente, es decir, que han participado en los procesos de acreditación y que presentan reclamos formales en el SERNAC en los años 2009 y 2010. Por tanto, el muestreo fue de carácter intencional.

\section{Variables}

Se trabajó con un indicador de la calidad institucional, medido a través de los años de acreditación institucional dados por la Comisión Nacional de Acreditación de Chile. Al mismo tiempo, se utilizó como un indicador inverso de la calidad del servicio una variable promedio de los índices de reclamos presentados durante los años 2009 y 2010. Dicho índice hace referencia al número de reclamos por cada 1.000 matriculados en la universidad, por año. De esta forma se trabajó con datos secundarios obtenidos desde la Comisión Nacional de Acreditación de Chile (ver www.cnachile.cl) y del Servicio Nacional del Consumidor (ver www.sernac.cl).

\section{Hipótesis de Trabajo}

A continuación se enuncian las hipótesis que fueron utilizadas:

\section{Hipótesis Conceptual}

Existe una relación entre el aseguramiento de la calidad institucional y la calidad del servicio de las universidades privadas chilenas. 


\section{Hipótesis Operacional}

Al aumentar el número de años de acreditación disminuye el índice de reclamos en las universidades privadas chilenas.

\section{Hipótesis Nula}

No existe una relación entre el aseguramiento de la calidad institucional y la calidad del servicio de las universidades privadas chilenas.

\section{Métodos}

Se realizó un análisis de regresión lineal simple, con el objetivo de analizar la relación entre ambas variables y el impacto de la variable independiente sobre la dependiente [9] con la siguiente ecuación:

$$
\text { Reclamos }=A+B * \text { Años de Acreditación }+E_{i}
$$

donde:

Reclamos: es la variable dependiente.

$A$ : es la constante de modelo que considera las variables omitidas por el mismo.

$B$ : es el factor de ponderación.

$E_{i}$ : el error residual del modelo.

\section{RESULTADOS}

A partir de los resultados obtenidos es posible señalar que sí existe una relación entre los años de acreditación de las universidades privadas y el índice de reclamos presentados contra éstas, apoyándose por tanto la hipótesis operacional $(\sigma=0,003)$. La relación entre ambas variables es de una fuerza media (R: 0,672). Los años de acreditación explican en un $41,5 \%$ ( $R^{2}$ ajustado) a la variable dependiente índice de reclamos. Ambas variables tienen una variación compartida de 0,45. La relación entre ambas variables es inversa, donde al aumentar los años de acreditación en las universidades privadas, disminuye el índice de reclamos en éstas. De esta manera, se puede apoyar la hipótesis operacional planteada en el estudio.

Esto último se puede constatar más claramente al analizar el coeficiente $B$, donde al aumentar en un año la acreditación de las universidades privadas, el índice de reclamos disminuye en $-1,1$ el índice de reclamos en las universidades.

Los resultados demuestran la relación existente entre la calidad de servicio y la calidad de aseguramiento de la calidad, reafirmando los planteamientos señalados con anterioridad, respecto a la importancia que tienen los procesos que aseguran y resguardan la calidad institucional de las universidades, permitiendo con eso que los usuarios tengan una buena percepción de la calidad de los servicios entregados.

En consonancia con los resultados obtenidos, es posible establecer que las universidades privadas también se preocupan de asegurar la calidad de sus instituciones, tal como ocurre en las universidades privadas del Medio Oriente, donde la calidad de servicio que le entregan a sus estudiantes es un aspecto crucial de resguardar, razón por la cual las oficinas de registro académicos son consideradas como elementos vitales para otorgar un buen servicio a los estudiantes [2].

De esta forma, se puede señalar que los factores identificados no difieren de los elementos que forman parte de los procesos del aseguramiento de la calidad. Este vínculo se puede establecer, al existir un consenso amplio de que la calidad de servicio es una actitud de juicio general sobre un servicio superior, aunque la naturaleza exacta de esta actitud es aún poco clara [1].

Complementando lo anterior, es importante señalar que los reclamos presentados por los estudiantes en el Servicio Nacional del Consumidor durante los años 2009 y 2010 hacen referencia a elementos tales como: el incumplimiento de las condiciones contratadas, la entrega de un servicio defectuoso, la falta de información oportuna y veraz, la facturación indebida, la mala calidad del servicio administrativo entre otras (SERNAC, 2009, 2010). Aspectos que se configuran como indicadores constituyentes de una deficiente calidad del servicio reflejado en el promedio de los índices de reclamos de los años 2009 y 2010.

En este sentido, el aseguramiento de la calidad puede ser descrito como el conjunto de actividades planeadas sistemáticamente para entregar la confianza suficiente de que los productos o servicios entregados están satisfaciendo los requerimientos de calidad [34].

Los resultados muestran además una gran dispersión de casos en torno a la línea de regresión, tal como se puede ver en la Figura 1. 


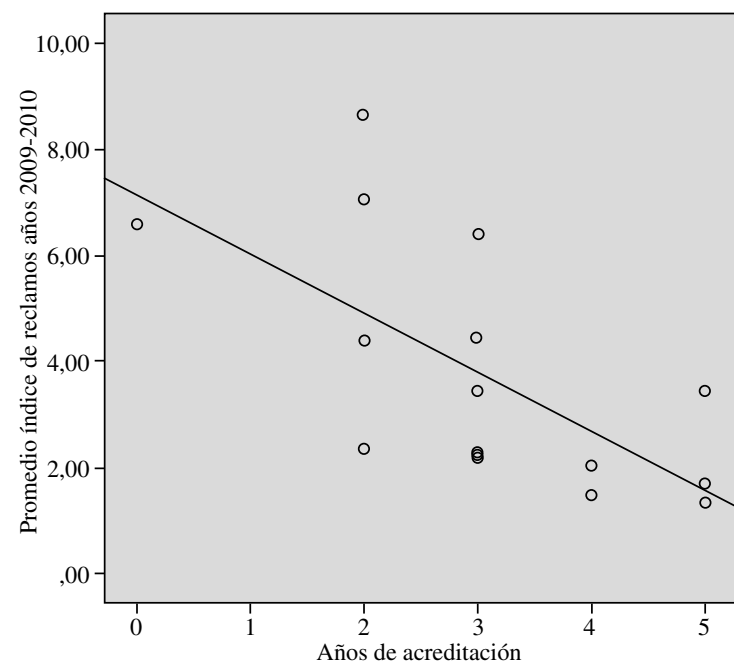

$\mathrm{R}^{2}$ Lineal $=0,452$

Figura 1. Regresión lineal entre años de acreditación e índice de reclamos.

Tabla 1. Resultados de la regresión lineal entre años de acreditación e índice de reclamos.

\begin{tabular}{|l|c|c|c|c|}
\hline Modelo & $\mathbf{R}$ & R cuadrado & $\begin{array}{c}\text { R cuadrado } \\
\text { corregida }\end{array}$ & $\begin{array}{c}\text { Error típ. de la } \\
\text { estimación }\end{array}$ \\
\hline 1 &, $672(a)$ &, 452 &, 415 & 1,74392 \\
\hline
\end{tabular}

a Variables predictoras: (Constante), años de acreditación.

Tabla 2. Coeficientes de la regresión lineal entre años de acreditación e índice de reclamos.

\begin{tabular}{|l|l|c|c|c|c|c|}
\hline \multirow{2}{*}{ Modelo } & & \multicolumn{2}{|c|}{$\begin{array}{c}\text { Coeficientes no } \\
\text { estandarizados }\end{array}$} & $\begin{array}{c}\text { Coeficientes } \\
\text { estandarizados }\end{array}$ & \multirow{2}{*}{$\begin{array}{c}\text { T } \\
\text { B }\end{array}$} & $\begin{array}{c}\text { Sig. } \\
\text { Error típ. }\end{array}$ \\
\cline { 3 - 7 } & & $\mathbf{B}$ & Error típ. & Beta & & \\
\hline \multirow{2}{*}{1} & (Constante) & 7,147 & 1,089 & & 6,562 &, 000 \\
\cline { 2 - 7 } & Años de acreditación & $-1,111$ &, 316 &,- 672 & $-3,517$ &, 003 \\
\hline
\end{tabular}

a Variable dependiente: Índice de reclamos que presentan las IES periodo enero-noviembre 2010.

\section{CONCLUSIONES}

Analizar la Educación Superior hoy en día implica no sólo considerar el contexto actual, marcado por una alta competitividad generada por el incremento de la oferta y demanda de la Educación Superior. En este complejo escenario, la calidad en la Educación Superior se manifiesta como un aspecto diferenciador entre las universidades, siendo un aspecto a considerar por los estudiantes para evaluar y decidir en qué institución universitaria estudiar.

El aseguramiento de la calidad de las Instituciones de Educación Superior se transforma en un tema clave para resguardar la calidad de servicio y lograr de esta forma una buena percepción y satisfacción por parte de los usuarios de éstos.

El fortalecimiento de estrategias y mecanismos que logren mejorar la percepción que tienen los clientes de la educación superior respecto a los servicios recibidos se convierte en un factor fundamental para asegurar los niveles de calidad de las Instituciones de Educación Superior.

Es relevante consignar que éste es un estudio exploratorio, cuyos resultados, a pesar de no ser extrapolables, contribuyen al acervo de conocimientos 
respecto a este tema, develando la importancia que tiene analizar el impacto de los procesos de aseguramiento de la calidad sobre diversos aspectos de las instituciones de educación superior; en este caso se presenta una primera aproximación, donde se muestra su influencia sobre la calidad del servicio en las universidades privadas. Resulta necesario, por tanto, extender los futuros análisis respecto a otras instituciones de educación terciaria, y a otros elementos para ver cómo influyen las estrategias de aseguramiento de la calidad implementadas sobre éstos.

De esta manera, Instituciones de Educación Superior con un sistema de aseguramiento de la calidad aseguran una buena calidad de servicio, lo cual se refleja en bajos índices de reclamos, transformándose de esta forma en un elemento crítico para conseguir y mantener la sustentabilidad de las universidades en el contexto actual.

\section{AGRADECIMIENTOS}

Esta investigación es la resultante de la ejecución del proyecto FONDECYT 1090116 de la Comisión Nacional de Investigación Científica y Tecnológica de Chile.

\section{REFERENCIAS}

[1] F. Abdullah. "HEdPERF versus SERVPERF: The quest for ideal measuring instrument of service". Quality Assurance in Education. Vol. 13, Issue 4, pp. 305-328. 2005.

[2] K. Abouchedid and R. Nasser. "Assuring quality service in higher education: registration and advising attitudes in a private university in Lebanon". Quality Assurance in Education. Vol. 10, Issue 4, pp.198-206. 2002.

[3] A. Abukari and T. Comer. "Delivering higher education to meet local needs in a developing context: the quality dilemmas". Quality Assurance in Education. Vol. 18, Issue 3, p. 191. 2010.

[4] R. Angell, T. Heffernan and P. Megicks. "Service quality in postgraduate education". Quality Assurance in Education. Vol. 16, Issue 3, pp. 236-254. 2008.

[5] P. Altbach, L. Reisberg and L. Rumbley. "Trends in global higher education. A report prepared for the UNESCO 2009 World Conference on Higher Education". The United Nations Educational. 2009.

[6] A. Ashworth and R. Harvey. "Assessing quality in further and higher education". Higher Education Policy Series. Vol. 24, PA: Jessica Kingsley Publishers. 1994.

[7] D. Báráňová-Č́iderová and P. Mayer. "Knowledge Transfer in Higher Education Quality Management: The Case of Germany and Slovakia". Studia commercialia Bratislavensia. Vol. 3, Issue 9, pp. 18-34. 2010.

[8] A. Baumann and J. Blythe. "Globalization of higher education in nursing". The online Journal of Issues in Nursing. Vol. $13 \mathrm{~N}^{\circ} 2.2008$.

[9] G. Briones. "Metodología de la investigación cuantitativa en las ciencias sociales". ARFO Editores e Impresores. Colombia. 2002.

[10] J.J. Brunner. "El proceso de Bologna en el horizonte latinoamericano: límites y posibilidades". Revista de Educación, Madrid. 2008.

[11] C. Chua. "Perception of Quality in Higher Education". Australian Universities Quality Forum (AUQF). Adelaide. Australia. July 7-9, 2004.

[12] CINDA. "Marco de referencia para el proyecto sobre aseguramiento de la calidad". Proyecto ALFA No DCI-ALA/2008/42. "Aseguramiento de la Calidad: políticas públicas y gestión universitaria". 2009.

[13] E. Cohen. "Educación, eficiencia y equidad: una difícil convivencia". Revista Iberoamericana de Educación. $\mathrm{N}^{\mathrm{o}}$ 030, pp. 105-124. Organización de Estados Iberoamericanos para la Educación, la Ciencia y la Cultura (OIE). Madrid, España. Septiembre-Diciembre 2002.

[14] B. Collis and J. Moonen. "Web 2.0 tools and processes in higher education: quality perspectives". Educational Media International. Vol. 45, Issue 2, pp. 93-106. 2008.

[15] D. Dill. "Quality Assurance in Higher Educacion: Practices and Issues". The 3rd International Encyclopedia of Education, Editors-in-Chief Barry McGaw, Eva Baker and Penelope P. Peterson, Elsevier Publications. 2007. 
[16] G. Doherty. "On quality in education". Quality Assurance in Education. Vol. 16, Issue 3, pp. 255-265. 2008.

[17] L. Dominelli and A. Hoogvelt. "Globalization, Contract Government and the Taylorization of Intellectual Labour in Academia”. Studies in Political Economy. Vol. 49, pp. 72-100. 2009.

[18] M. Edwards, E. Tovar and L. Sánchez-Ruiz. "Strengths and obstacles for quality assurance in the European Higher Education Area: the Spanish case". International network for engineering education and research. 2009.

[19] P. Ewell. "Twenty Years of Quality Assurance in Higher Education: What's Happened and What's Different?" Quality in Higher Education. Vol. 16, Issue 2, pp. 173-175. 2011.

[20] H. Fitri, A. Ilias, R. Rahman and M. Zulkeflee. "Service quality and student satisfaction: A case study at private higher educations institutions". International Business Research. Vol. 1, Issue 3, pp. 163-175. 2008.

[21] J. Gallifa and P. Batallé. "Student perceptions of service quality in a multi-campus higher education system in Spain". Quality Assurance in Education Vol. $18 \mathrm{~N}^{\circ}$ 2, pp. 156-170. 2010.

[22] A. Jalali, A. Islam and K. Halim. "Service Satisfaction: The case of a higher learning institution in Malaysia”. International Education Studies. Vol. 4, Issue 1, pp. 182192. 2011.

[23] Ji-Y. Jang. "Analysis of the relationship between internationalization and the quality of higher education". A dissertation submitted to the Faculty of the Graduate School of the University of Minnesota. In partial fulfillment of the requirements for the degree of Doctor of Philosophy. 2009.

[24] J. Kettunen. "A conceptual framework to help evaluate the quality of institutional performance". Quality Assurance in Education. Vol. $16 \mathrm{~N}^{\circ}$ 4, pp. 322-332. 2008.

[25] M. Kwiek. "Globalization and Higher Education". Higher Education in Europe. Vol. 26, Issue 1. 2001.

[26] M. Malik, R. Danish and A. Usman. "The impact of service quality on students' satisfaction in higher education Institutes of Punjab". Journal of Management Research. Vol. 2, Issue 2, pp. 1-11. 2010.
[27] L. Morley. "Opportunity or exploitation? Women and quality assurance in higher education". Gender and Education. Vol. 17, Issue 4, pp. 411-429. 2005.

[28] D. Neubauer. "Globalization and education. Characteristics, dynamics, implications". En P. Hershock, M. Mason, Mark and J. Hawkings (editors). "Changing Education leadership innovation and development in globalizing Asia Pacific". Comparative Education Research Centre. University of Hong Kong. 2007.

[29] C. Ogbodo and N. Nwaoku. "Quality Assurance in Higher Education". Towards Quality in African Higher Education. 2007.

[30] O. Onocha. "Quality Assurance in Teacher Education" A Discussion Paper Presented at the NTI'S 25th Anniversary Celebration Kaduna. 2002.

[31] A. Parasuraman, V.A. Zeithaml and L.L. Berry. "A conceptual model of service quality and its implications for future research", Journal of Marketing, Vol. 49, pp. 41-50. 1985.

[32] W. Patrick and E. Stanley. "Identifyng excellence in undergraduate education: are we even close?". Change. Vol. 33, Issue 3, pp. 19-23. 1998.

[33] R. Quintero, L. Cabrera y P. Martínez. "Diagnóstico de expectativas y calidad del servicio en los estudiantes del programa ingeniería industrial de la Universidad de Magdalena". Tesis para optar al grado de Magíster en Administración de Empresas. Universidad del Norte. Chile. 2009.

[34] N. Reid. "Quality assurance in higher education in Pakistan looking to the future". 3rd International Conference in Assessing Quality in Higher Education. Lahore, Pakistan. December 6-8, 2010.

[35] E. Rodríguez. "El rol de las universidades en la sociedad del conocimiento y en la era de la globalización: evidencia desde Chile". Interciencia. Vol. $34 \mathrm{~N}^{\circ}$ 11. 2009.

[36] G. Soutar and M. McNeil. "Measuring service quality in a tertiary institution", Journal of Educational Administration, Vol. 34, Issue 1, pp. 72-82, 1996.

[37] B. Stensaker. "Outcomes of Quality Assurance: A Discussion of Knowledge, Methodology and Validity". Quality in Higher Education. Vol. 14, Issue 1, pp. 3-13. 2008. 
[38] M. Tsiniduo, V. Gerogiannis and P. Fitsilis. "Evaluation of the factors that determine quality in higher education: an empirical study". Quality Assurance in Education. Vol. 18, Issue 3, pp. 227-244. 2010.

[39] R. Voss, T. Gruber and I. Szmigin. "Service quality in higher education: The role of student expectations". Journal of Business Research. Vol. 60, pp. 949-959. 2007.
[40] L. Wood, T. Thomas and B. Rigby. "Assessment and standards for graduate outcomes". Assian Social Science. Vol. 7, Issue 4, pp. 12-17. 2011.

[41] L.Yao. "The present situation and development tendency of higher education quality evaluation in western countries". Private Education Research. Issue 2006-03. 2006. DOI: CNKI:SUN: MBJY.0.2006-03-010. 